\title{
Cosmic-ray spectra of primary protons and high altitude muons deconvolved from observed atmospheric gamma rays
}

\author{
K. Yoshida \\ Department of Electronic Information Systems, Shibaura Institute of Technology, Saitama, Japar * \\ R. Ohmori and Y. Sato \\ Utsunomiya University, Utsunomiya, Japan \\ T. Kobayashi \\ Department of Physics and Mathematics, Aoyama Gakuin University, Sagamihara, Japan \\ Y. Komori \\ Kanagawa University of Human Services, Yokosuka, Japan \\ J. Nishimura \\ Institute of Space and Astronautical Science, Sagamihara, Japan
}

(Dated: May 21, 2018)

\begin{abstract}
We have observed atmospheric gamma rays from $30 \mathrm{GeV}$ to $8 \mathrm{TeV}$, using emulsion chambers at balloon altitudes, accumulating the largest total exposure in this energy range to date, $S \Omega T \sim 6.66 \mathrm{~m}^{2} \mathrm{sr}$ day. At very high altitudes, with residual overburden only a few $\mathrm{g} \mathrm{cm}^{-2}$, atmospheric gamma rays are mainly produced by a single interaction of primary cosmic rays with overlying atmospheric nuclei. Thus, we can use these gamma rays to study the spectrum of primary cosmic rays and their products in the atmosphere. From the observed atmospheric gamma ray spectrum, we deconvolved the primary cosmic-ray proton spectrum, assuming appropriate hadronic interaction models. Our deconvolved proton spectrum covers the energy range from $200 \mathrm{GeV}$ to $50 \mathrm{TeV}$, which fills a gap in the currently available primary cosmic-ray proton spectra. We also estimated the atmospheric muon spectrum above $30 \mathrm{GeV}$ at high altitude from our gamma-ray spectrum, almost without reference to the primary cosmic rays, and compared the estimated flux with direct muon observations below $10 \mathrm{GeV}$.
\end{abstract}

PACS numbers: 92.60.hx, 13.85.Tp

\section{INTRODUCTION}

The primary cosmic-ray proton spectrum is one of the most important quantities needed to interpret cosmic-ray phenomena inside the atmosphere. Many observations of primary protons have been performed since the discovery of cosmic rays. Recently, proton flux measurements have attracted renewed attention, since they are needed to precisely estimate the absolute flux of atmospheric neutrinos, providing critical input for analyses of neutrino oscillations being performed with Super-Kamiokande [1] and similar detectors. In particular, atmospheric neutrinos with higher energies, $\sim 100 \mathrm{GeV}$, which are detected as upward through-going muons, are mainly produced from the interaction of $\sim 1 \mathrm{TeV}$ primary protons in the atmosphere. Similarly important are observations of the absolute flux of muons, which are the partners of neutrinos, and thus provide a check on atmospheric neutrino flux estimates. Since atmospheric gamma rays at high altitude, with residual overburden of a few $\mathrm{g} \mathrm{cm}^{-2}$, are mainly produced by a single interaction of primary cos-

\footnotetext{
*Electronic address: yoshida@shibaura-it.ac.jp
}

mic rays with overlying atmospheric nuclei, we can use these gamma rays to study the spectrum of primary cosmic rays and their products in the atmosphere.

We have been observing primary cosmic ray electrons at balloon altitudes using emulsion chambers for many years, and successfully obtained the energy spectrum of electrons in the energy range from $30 \mathrm{GeV}$ to $3 \mathrm{TeV}[2,3]$. In the course of these electron observations, we have simultaneously observed atmospheric gamma rays. In particular, since gamma rays produce electromagnetic showers just like electrons, emulsion chambers are not only suitable to observe the gamma rays in the atmosphere, but measurement of the gamma ray showers is a necessary part of the electron analysis, required to check and calibrate the performance of the emulsion chambers.

Atmospheric gamma rays have been observed by the BETS group in the energy range from a few $\mathrm{GeV}$ to several $10 \mathrm{GeV}$ at mountain altitude $(2.77 \mathrm{~km})$ and at balloon altitudes $(15-32 \mathrm{~km})[4]$. They found that hadronic interaction simulation codes such as the Lund group's Fritiof V7.02, or Dpmjet3 give results fairly consistent with the observed energy spectra and altitude variation of the gamma-ray flux, within the accuracy of their measurements. The JACEE group also observed high-energy gamma rays in the $3-30 \mathrm{TeV}$ range at overburden 
$\sim 5.5 \mathrm{~g} \mathrm{~cm}^{-2}$ for $120-150 \mathrm{hr}[5]$.

Atmospheric neutrinos, muons, and gamma rays are decay products of pions produced in the interaction of primary cosmic rays with atmospheric nuclei:

$$
\begin{aligned}
p+N & \rightarrow \pi^{ \pm} \rightarrow \mu^{ \pm}+\nu_{\mu}\left(\bar{\nu}_{\mu}\right) \\
& \searrow \pi^{ \pm} \rightarrow 2 \gamma
\end{aligned}
$$

In addition to pions, $K$ and $\eta$ mesons are produced in the primary interactions, and also decay into muons and gamma rays, making minor contributions to the net fluxes.

Atmospheric gamma rays are mainly the decay products of $\pi^{0}$. When we observe atmospheric gamma rays at very high altitudes, corresponding to residual overburdens of several $\mathrm{g} \mathrm{cm}^{-2}$, the thickness of atmosphere above the detector is $\sim 0.05$ nuclear mean free path or $\sim 0.1$ radiation lengths (r.l.). Thus, hadronic cascades and electromagnetic showers cannot develop significantly in the atmosphere before reaching the detector. The observed gamma rays are almost all produced by the first interaction of primary cosmic rays (protons, helium and heavier nuclei) with atmospheric nuclei. Therefore, using the appropriate hadronic interaction models, we can reliably deconvolve the primary proton flux from the observed spectrum of atmospheric gamma rays produced by $\pi^{0}$. This can be done in a semi-analytic way, because of the approximate scaling nature of the pion production cross section. In this deconvolution, we include the effects of contributions from primary helium and heavier nuclei, and also the minor contribution of gamma rays from decays of $\eta$ and $K$ mesons.

The proton spectra have been measured with two kinds of detectors: magnetic spectrometers and calorimeters. Although magnetic spectrometers have excellent energy resolution, their maximum observable energy is limited by the maximum detectable rigidity (MDR) to $\sim 1 \mathrm{TeV}$. The BESS, AMS-01 and CAPRICE instruments are typical detectors using magnetic spectrometers, and have precisely measured primary proton spectra up to $\sim 100 \mathrm{GeV}[6,6,8]$. BESS and AMS-01 give results consistent with each other within an error of a few $\%$, while CAPRICE reports a $10 \%$ lower proton flux. Recently, the BESS-TeV spectrometer, with improved MDR [9], observed primary proton spectra up to $540 \mathrm{GeV}$. As for calorimeters, electronic detectors using scintillators, as well as passive emulsion chambers have been used to observe the proton spectrum in the higher energy region. The first measurements of the proton spectrum in the $\mathrm{TeV}$ region were made by the PROTON satellite-borne calorimeters 10]. These observations were followed by an ionization spectrometer flown at balloon altitudes whose energy range extend from $50 \mathrm{GeV}$ to $2 \mathrm{TeV}$ [1]. The proton spectrum in the energy range from $10 \mathrm{TeV}$ to $1000 \mathrm{TeV}$ has been measured by the JACEE Collaboration and RUNJOB Collaborations using emulsion cham- ber detectors 12, 13, 14, and references therein]. Recently, ATIC, which is a calorimeter with BGO scintillators, observed primary proton spectrum in $30 \mathrm{GeV}$ $50 \mathrm{TeV}$ [15]. However, more accurate measurements of the proton spectrum are still required in the energy range from $100 \mathrm{GeV}$ to $10 \mathrm{TeV}$.

In detectors that directly observe primary cosmic rays, such as JACEE and RUNJOB, the energies of primary protons are estimated from electromagnetic showers produced by nuclear interactions within the emulsion chambers. Since the electromagnetic shower developed in the chamber is actually initiated by multiple secondary gamma rays, and its structure is affected by their emission angles, energy estimation for the primary particle is complicated. On the other hand, in the present work, we derive the energy of primary protons from observed electromagnetic showers initiated by one single gamma ray, so that the estimation of proton fluxes from atmospheric gamma rays is simpler and therefore more reliable.

As a first approximation, the number of charged pions produced in hadronic interactions is almost two times of neutral pions. Hence we can estimate the production rate of charged pions from atmospheric gamma rays. Since the muons are mostly produced from the decay of charged pions, we can also directly estimate the muon flux without reference to the primary cosmic ray flux, correcting for the minor contribution of $\eta$ and $K$ mesons. More precisely, widely accepted hadronic interaction models are used to derive the muon spectrum from the gamma-ray spectrum.

The MASS, CAPRICE, HEAT and BESS group have performed direct atmospheric muon observations at various balloon altitudes [16, 17, 18, 19, 20]. The muon spectrum at various atmospheric depths gives important information to check the reliability of calculations performed to estimate the neutrino flux 21]. However, the muon flux at large depths depends on the detailed structure of atmospheric density as a function of altitude, which is subject to seasonal variation and meteorological conditions. This brings complications and ambiguities into the muon flux estimation. On the other hand, the flux of atmospheric gamma rays at stratospheric altitudes depends only on the residual atmospheric overburden, which can be estimated much less ambiguously than the muon flux. Hence, an accurate measurement of the gamma-ray flux can be also used to calibrate the neutrino flux calculations, because of the relation between gamma-ray and neutrino production in the atmosphere as shown in the process (11).

In this paper, we present measurements of the atmospheric gamma-ray spectrum due to hadronic interactions in the energy range of $30 \mathrm{GeV}$ to $8 \mathrm{TeV}$, observed at balloon altitudes with emulsion chambers. We also present results from the deconvolution of primary cosmic-ray proton and high altitude muon spectra from our gamma-ray observations. 


\section{ATMOSPHERIC GAMMA-RAY OBSERVATION}

\section{A. Balloon observations}

We have observed primary electrons with balloonborne emulsion chambers in many flights between 1968 and 2001 [2, 3, 22]. Simultaneously, we have also observed atmospheric gamma-rays to check the performance of the emulsion chambers in each balloon experiment. The pressure altitude records for each flight correspond to residual atmospheric overburdens in the range from $4.0 \mathrm{~g} \mathrm{~cm}^{-2}$ to $9.4 \mathrm{~g} \mathrm{~cm}^{-2}$. The total cumulative effective exposure $S \Omega T$ for gamma rays is $6.66 \mathrm{~m}^{2}$-sr-day, which is larger than any other atmospheric gamma-ray observations performed at balloon altitudes in the energy range of $30 \mathrm{GeV}$ to $8 \mathrm{TeV}$. In Table【 we summarize the series of experiments since 1968. Nishimura et al. (1980) have reported the spectra of primary electrons and atmospheric gamma rays for the 1968-1976 observations in our previous work [2].

\section{B. Detector}

Emulsion chambers consist of nuclear emulsion plates and lead plates, which are stacked alternately. Nuclear emulsion plates sample the development of electromagnetic showers produced in the lead plates. X-ray films are also inserted to allow rapid, naked-eye scanning for high energy showers, which produce dark spots in the film. The threshold energy for shower detection depends on the background and the sensitivity of the films; some details of the detection threshold for various films are described later. Figure 1 shows a typical emulsion chamber configuration. Detailed configurations and performance are described in Nishimura et al. (1980) [2] and Kobayashi et al. (1999) [3].

In emulsion chambers, it is possible to measure the location of shower tracks in each emulsion plate with a precision of $\sim 1 \mu \mathrm{m}$. Because of this high position resolution, we can inspect the shower starting points in detail and unambiguously distinguish showers due to electrons, gamma rays, and other hadronic interaction events [2]. By inspecting various specific features of those events, the rejection power for protons misidentified as electron candidates is found to be as large as $10^{5}[3]$.

We measure the shower particles within a circle of $100 \mu \mathrm{m}$ radius from shower axis, which means that we select the shower particles with higher energy, which have suffered less multiple scattering in the chamber. Hence, the number of the shower particles drops off faster than for all shower particles, and the shower maximum appears in $\sim 6$ r.l. for $1 \mathrm{TeV}$ electrons, while the maximum of the total number of shower particles appears in $\sim 12$ r.l. for $1 \mathrm{TeV}$ electrons. This means that we can determine the energy of higher energy incident electrons with thinner detector. The typical size and thickness of the detector are $40 \mathrm{~cm} \times 50 \mathrm{~cm}$, and $8 \mathrm{~cm}(\sim 9$ r.l. $)$, respectively. Thus the emulsion chamber has the advantage of a large effective area combined with a wide field of view compared with other detectors. Because of the simple configuration of the detector, we can estimate the geometrical factor $(S \Omega)$ very accurately, a task which is difficult for some electronic detectors.

For electron observations, the effective geometrical factor is given by

$$
S \Omega_{e}=2 \pi S \eta \int_{0}^{\theta_{0}} \cos \theta \sin \theta d \theta=\pi S \eta \sin ^{2} \theta_{0},
$$

where $\theta_{0}$ is the upper limit of incident angles and $\eta$ is the efficiency of events that pass through the top and bottom emulsion plates, the so-called "edge effect". On the other hand, for atmospheric gamma-ray observations, the effective geometrical factor is given by

$$
S \Omega_{\gamma}=2 \pi S \eta \int_{0}^{\theta_{0}} \sin \theta d \theta=2 \pi S \eta\left(1-\cos \theta_{0}\right) .
$$

Here, we corrected $S \Omega_{\gamma}$ to obtain the vertical flux, because the gamma rays have a $1 / \cos \theta$ enhancement relative to isotropic primary cosmic rays, proportional to the path length in the overlying atmosphere at balloon altitudes. Figure 2 shows the zenith angle distribution observed with emulsion chambers, compared with expectation calculated taking into account small corrections due to the elongation of the path length in the overlying air for large zenith angles, which affect the amount of absorption of the electrons and gamma rays. In the typical case of $\theta_{0}=60^{\circ}$ and $S=0.40 \times 0.50 \mathrm{~m}^{2}, S \Omega_{\gamma}$ is $0.52 \mathrm{~m}^{2}$ sr with $\eta$ of 0.82 for the chamber thickness of $8.0 \mathrm{~cm}$. For the comparison of emulsion chambers with other detectors, we summarize the effective geometrical factor $S \Omega_{e}$ with efficiency for primary electron experiments in Table We can see how the emulsion chamber is efficient for observation of low-flux components of cosmic rays such as primary electrons and atmospheric gamma rays.

\section{Scanning method}

Since high energy electro-magnetic showers above several $100 \mathrm{GeV}$ leave dark spots on X-ray films, we can detect these showers with the naked eye by scanning the $\mathrm{X}$-ray films. We locate the corresponding tracks in the adjacent emulsion plate using microscopes, trace them back through the stack to the cascade starting point, and identify the incoming particle. The validity of particle identification in our experiments is also checked by comparison with the expected zenith angle distributions and shower starting point distributions, as described in our previous work [2, 3]. We picked up events with zenith angle less than $60^{\circ}$. The detection threshold of the X-ray film, although it depends on the accumulated background tracks and fog in the film, is $\sim 500 \mathrm{GeV}$ for Sakura type-N 
TABLE I: List of balloon flights

\begin{tabular}{lrrccr}
\hline \hline Flight & $\begin{array}{c}\text { Area } \\
\left(\mathrm{m}^{2}\right)\end{array}$ & $\begin{array}{c}\text { Time } \\
(\mathrm{min})\end{array}$ & $\begin{array}{r}\text { Average Altitude } \\
\left(\mathrm{g} \mathrm{cm}^{-2}\right)\end{array}$ & $\begin{array}{l}S \Omega_{\gamma} T^{*} \\
\left(\mathrm{~m}^{2} \mathrm{sr} \mathrm{s}\right)\end{array}$ & Launch Site \\
\hline 1968 & 0.05 & 380 & 6.1 & $2.381 \times 10^{3}$ & Harunomachi, Japan \\
1969 & 0.05 & 267 & 7.1 & $1.799 \times 10^{3}$ & Harunomachi, Japan \\
1970 & 0.05 & 1136 & 6.1 & $7.900 \times 10^{3}$ & Sanriku, Japan \\
1973 & 0.20 & 833 & 8.2 & $2.694 \times 10^{4}$ & Sanriku, Japan \\
1976 & 0.40 & 1526 & 4.0 & $9.425 \times 10^{4}$ & Palestine, USA \\
1977 & 0.78 & 1760 & 4.5 & $6.549 \times 10^{4}$ & Palestine, USA \\
1979 & 0.80 & 1680 & 4.9 & $1.411 \times 10^{5}$ & Palestine, USA \\
1980 & 0.80 & 2029 & 7.8 & $1.099 \times 10^{5}$ & Palestine, USA \\
1984 & 0.20 & 576 & 9.2 & $7.106 \times 10^{3}$ & Sanriku, Japan \\
1985 & 0.40 & 940 & 9.4 & $1.324 \times 10^{4}$ & Sanriku, Japan \\
1988 & 0.20 & 647 & 7.1 & $3.929 \times 10^{3}$ & Uchinoura, Japan \\
1996 & 0.20 & 2092 & 4.6 & $6.497 \times 10^{4}$ & Sanriku, Japan \\
1998 & 0.20 & 1178 & 5.6 & $3.638 \times 10^{4}$ & Sanriku, Japan \\
2001 & 0.20 & 1108 & 5.5 & $1.096 \times 10^{2}$ & Sanriku, Japan \\
\hline \multirow{*}{*}{ Effective }
\end{tabular}

* Effective $S \Omega_{\gamma} T$ for the gamma-ray observations.

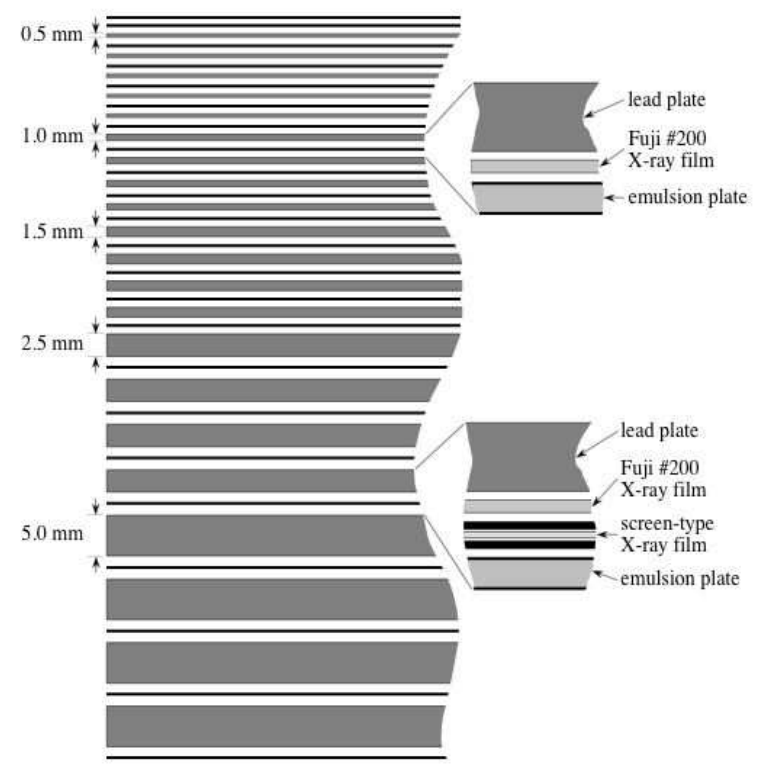

FIG. 1: Typical configuration of the emulsion chamber in cross-sectional drawing from side view.

X-ray film used before 1984, 800 GeV for Fuji \#200 Xray film and $\sim 200 \mathrm{GeV}$ for screen type $\mathrm{X}$-ray films such as Fuji G8-RXO, G12-RXO, HR8-HA30, HR12-HA30 used on and after 1984 [28].

To detect the electro-magnetic showers below a few hundred $\mathrm{GeV}$, the emulsion plates had to be directly scanned using microscopes. Although the emulsion chamber is the only detector which has succeeded in observing cosmic-ray electrons and gamma rays in the $\mathrm{TeV}$ region, it was difficult to observe electrons or gamma-rays below a few hundred $\mathrm{GeV}$ because of the tedious work

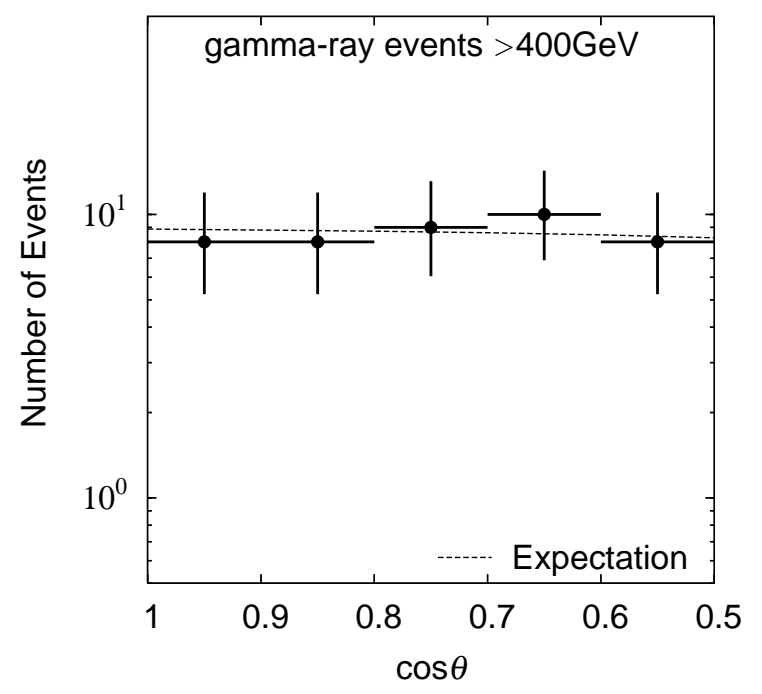

FIG. 2: Zenith angle distribution for atmospheric gamma rays beyond $400 \mathrm{GeV}$.

required in manual microscope scanning of the emulsion plates. In order to overcome this difficulty tracing low energy showers, in 2001 we started to apply the automatic scanning method which has been successfully developed by the Nagoya group to observe $\nu_{\tau}$ events with nuclear emulsions 29]. A fully automatic emulsion scanning system developed by the Nagoya group, called "Track Selector", consists of a motor-driven microscope XYZ stage with a controller, a fast read-out CCD and special hardware for 3D image processing,. It takes the images of 16 "slices", or layers within an emulsion plate, each of $512 \times 512$ pixels, corresponding to a field of view of $147 \times 106 \mu \mathrm{m}^{2}$, and then outputs position and angle data 
TABLE II: Examples of effective geometrical factors with efficiency of the electron detectors.

\begin{tabular}{lcr}
\hline \hline Detector & $S \Omega_{e}\left(\mathrm{~m}^{2} \mathrm{sr}\right)$ & reference \\
\hline T.R.D. & 0.11 & {$[23]$} \\
MASS-91 & 0.018 & {$[24]$} \\
CAPRICE94 & 0.016 & {$[25]$} \\
HEAT & 0.012 & {$[26]$} \\
BETS & 0.032 & {$[27]$} \\
AMS-01 & 0.10 & {$[7]$} \\
ECC & $0.38^{*}$ & {$[2]$} \\
${ }^{*} S \Omega_{e}$ in the typical emulsion chamber.
\end{tabular}

for each track found in the emulsion plate. It takes approximately $16 \mathrm{hr}$ to perform general scanning over an area of $1 \mathrm{~cm}^{2}$. We scan the emulsion plate at $3 \mathrm{r} .1$. depth with the Track Selector, and read out all tracks with $\tan \theta<0.3$. Shower candidates in the track data are identified with off-line programs. Selected shower candidates are traced back to the top plate of the chamber by searching for tracks having the predicted angle and position. We carefully inspect the starting behavior of a shower to see whether it was initiated by an electron, gamma ray or hadron. For the balloon detectors in 2001, we successfully observed atmospheric gamma rays down to $30 \mathrm{GeV}$ using this automatic scanning system.

\section{Energy determination}

Shower energy was determined by comparing the number of shower tracks at various depths with the theoretical transition curves, and fitting to the integrated track length, used to estimate total ionization in the cascade. As the chamber structure is slightly different for each flight, we calculated the shower development for each chamber using a Monte Carlo simulation code called EPICS [30]. Results calculated using the EPICS code were confirmed by emulsion chambers exposed to FNAL electron beams [2] in which showers of $100 \mathrm{GeV}$ electrons were re-analyzed. Figure 3 4 show longitudinal development of the average number of shower electrons, and energy distribution of the FNAL experimental and simulated data. The simulations well represent the experimental data, and the determined energy with the simulation for $100 \mathrm{GeV}$ electrons is consistent with the experiment within a precision of a few \%. The energy resolution is $12 \%$ at $100 \mathrm{GeV}$, as shown in Fig. 4. The energy resolutions for each emulsion chamber are well represented by the form of

$$
\frac{\sigma}{E_{0}}=\left[a^{2}\left(\frac{E_{0}}{100 \mathrm{GeV}}\right)^{-1}+b^{2}+c^{2}\left(\frac{E_{0}}{100 \mathrm{GeV}}\right)\right]^{1 / 2}
$$

where $E_{0}$ is the incident gamma-ray energy and $\sigma$ is the standard deviation of energy determination. The first term in right-hand side root represents statistics-related fluctuations of the number of shower particles, while the last term represents fluctuations due to "punch-through", shower particles escaping from the finite thickness of the detector. The adjustable coefficients of $a, b$, and $c$ are derived for each chamber using the results of the Monte Carlo simulations. Figure [5] shows some examples of the energy dependence of energy resolution for incident gamma rays from the simulations, whose showers start from pair electrons. The fitted functions of (4) are also plotted. The coefficients of $a, b$, and $c$ typically have the values of $a \simeq 12-13 \%, b \simeq 5-10 \%$, and $c \simeq 2-3 \%$.

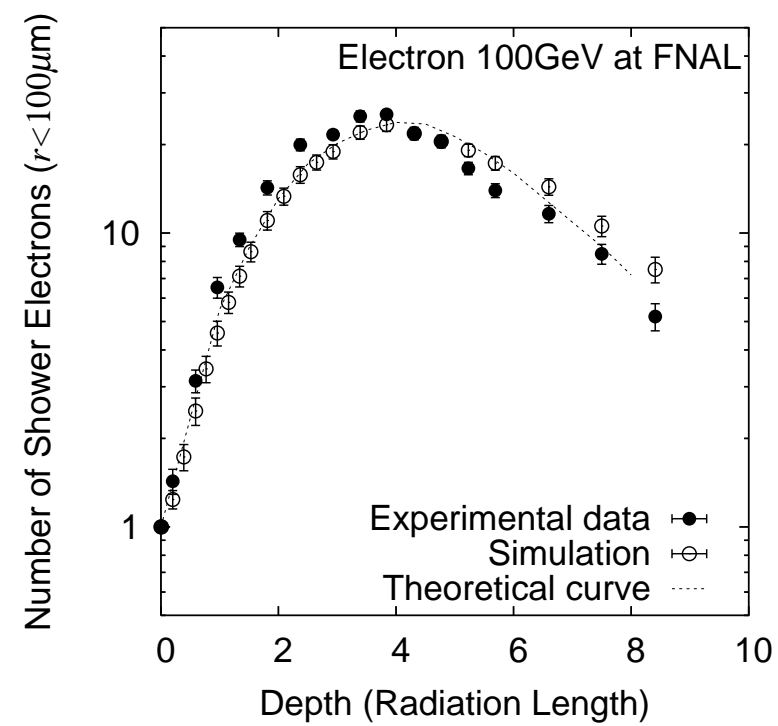

FIG. 3: Longitudinal development of the averaged number of shower electrons within a radius of $100 \mu \mathrm{m}$. Error bars show the uncertainties of the means. Theoretical curve is taken from Nishimura et al. (1980) [2].

\section{Atmospheric gamma-ray spectrum}

We observed atmospheric gamma rays at each balloon altitude, and derived the vertical gamma-ray spectrum normalized at $4.0 \mathrm{~g} \mathrm{~cm}^{-2}$ originated from hadronic interactions using the following formula:

$$
\begin{aligned}
J_{\gamma}(E)=\left(\frac{N_{\gamma}}{S \Omega_{\gamma} T \Delta E C_{\mathrm{eff}} C_{\mathrm{enh}}}-C_{\mathrm{brem}}\right) \cdot C_{\mathrm{alt}} \\
\left(\mathrm{m}^{-2} \mathrm{~s}^{-1} \mathrm{sr}^{-1} \mathrm{GeV}^{-1}\right) .
\end{aligned}
$$

Here $N_{\gamma}$ is the number of gamma-ray events, $C_{\text {eff }}$ is gamma-ray detection efficiency, $C_{\text {enh }}$ is enhancement factor due to the energy resolution as described below, $C_{\text {brem }}$ is bremsstrahlung gamma-ray flux from primary electrons, and $C_{\text {alt }}$ is an altitude conversion factor to $4.0 \mathrm{~g} \mathrm{~cm}^{-2}$. $S \Omega_{\gamma}$ is the geometrical factor to obtain the vertical flux. 


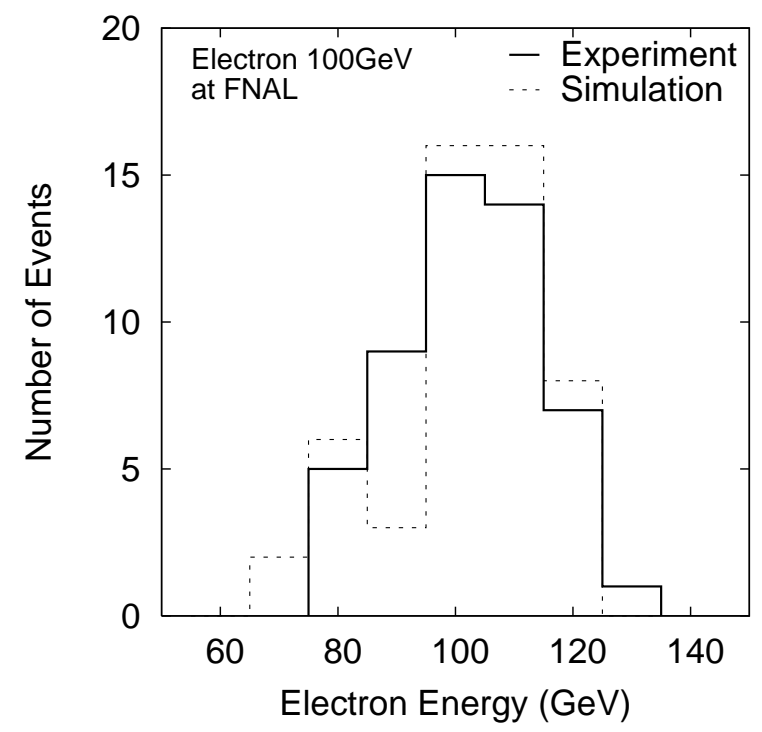

FIG. 4: Energy distributions of the simulated and experimental data for $100 \mathrm{GeV}$ electron beams at FNAL.
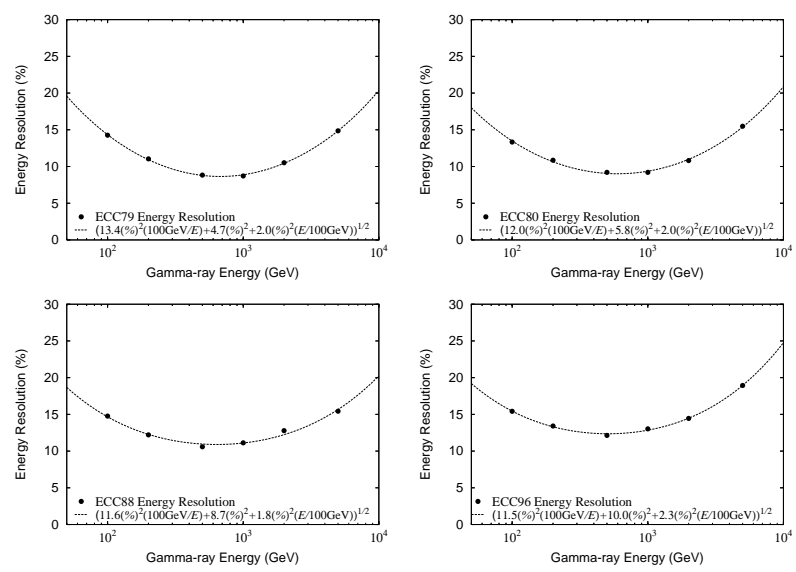

FIG. 5: Examples of the energy dependence of energy resolutions with the emulsion chambers for gamma rays from the simulations. The dash lines show the fitted function of (4).

Gamma-ray detection efficiency $C_{\text {eff }}$ in the chamber is given by

$$
C_{\text {eff }}=1-\exp \left(-\sigma_{0} T_{c}\right),
$$

where $\sigma_{0}=0.7733$ is the probability of pair creation in one radiation length, $T_{c}$ is the threshold depth of shower starting points for gamma rays. $C_{\text {eff }}$ ranges from 0.902 to 0.955 for our observations, corresponding to $T_{c}=3.0-$ 4.0 r.l. in the threshold depth.

The uncertainty of the energy determination has the effect of enhancing the absolute flux of gamma rays, in particular, for the steep power-law spectrum. We derived the enhancement factor $C_{\text {enh }}$ due to the energy resolution for each chamber, which ranges from 1.00 to 1.06 depending on gamma-ray energies and emulsion chamber

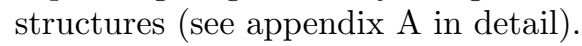

In this atmospheric gamma-ray spectrum, we subtracted the bremsstrahlung gamma rays produced by primary cosmic-ray electrons. The observed primary electron spectrum $J_{e}(E)$ is well represented by

$$
\begin{aligned}
& J_{e}(E)=1.6 \times 10^{-4}(E / 100 \mathrm{GeV})^{-3.3} \\
& \left(\mathrm{~m}^{-2} \mathrm{~s}^{-1} \mathrm{sr}^{-1} \mathrm{GeV}^{-1}\right)
\end{aligned}
$$

in the energy range of $30 \mathrm{GeV}-1 \mathrm{TeV}[2]$. At a depth of $x \mathrm{~g} \mathrm{~cm}^{-2}$, the bremsstrahlung gamma-ray spectrum from the electrons with a power-law index of -3.3 is given by

$$
\begin{aligned}
C_{\text {brem }}=J_{e}(E) C(s & =2.3) \\
& \times \frac{e^{-\sigma_{0} x / X_{0}}-e^{-A(s=2.3) x / X_{0}}}{A(s=2.3)-\sigma_{0}},
\end{aligned}
$$

where the radiation length in the atmosphere $X_{0}=$ $36.7 \mathrm{~g} \mathrm{~cm}^{-2}, A(s=2.3)=1.674$, and $C(s=2.3)=$ 0.4118. The notations $A$ and $C$ refer to the functions used in electro-magnetic shower theory, defined in reference 31.

The flux of the Galactic diffuse gamma-ray emission measured by EGRET is less than $0.1 \%$ of the atmospheric gamma rays at depths of several $\mathrm{g} \mathrm{cm}^{-2}$ [32]. Sreekumar et al. (1998) 33] reported that extra-galactic diffuse gamma rays observed with EGRET have a powerlaw spectrum with an index of -2.1 in the $50 \mathrm{MeV}$ to $100 \mathrm{GeV}$. Their flux at $100 \mathrm{GeV}$ corresponds to $\sim 0.5 \%$ of the atmospheric gamma rays, and there are also arguments that the flux of extra-galactic diffuse gamma rays is lower than their derivation [34]. Therefore, we ignored the contribution of the flux of astrophysical gamma rays to obtain the atmospheric gamma-ray flux.

The gamma-ray fluxes are normalized to $4.0 \mathrm{~g} \mathrm{~cm}^{-2}$ equivalent altitude from each observation altitude of $x \mathrm{~g} \mathrm{~cm}^{-2}$ using

$$
C_{\mathrm{alt}}=\frac{e^{-4.0 / \Lambda_{p}}-e^{-4.0 \sigma_{0} / X_{0}}}{e^{-x / \Lambda_{p}}-e^{-x \sigma_{0} / X_{0}}}
$$

where $\Lambda_{p}$ is an attenuation length of protons in the atmosphere, whose value is $\sim 100 \mathrm{~g} \mathrm{~cm}^{-2}$ in the $\mathrm{TeV}$ region as described in section

The total number of observed gamma rays is 330 events in the energy range from $30 \mathrm{GeV}$ to $8 \mathrm{TeV}$. After the corrections described, we derived the vertical spectrum of atmospheric gamma rays. Figure 6 shows the observed spectrum of atmospheric gamma-rays normalized at an altitude of $4.0 \mathrm{~g} \mathrm{~cm}^{-2}$, which is well represented by

$$
\begin{array}{r}
J_{\gamma}(E)=(1.12 \pm 0.13) \times 10^{-4}(E / 100 \mathrm{GeV})^{-2.73 \pm 0.06} \\
\left(\mathrm{~m}^{-2} \mathrm{~s}^{-1} \mathrm{sr}^{-1} \mathrm{GeV}^{-1}\right) .
\end{array}
$$

The flux values and numbers of the gamma rays in each energy bin are listed in Table III 


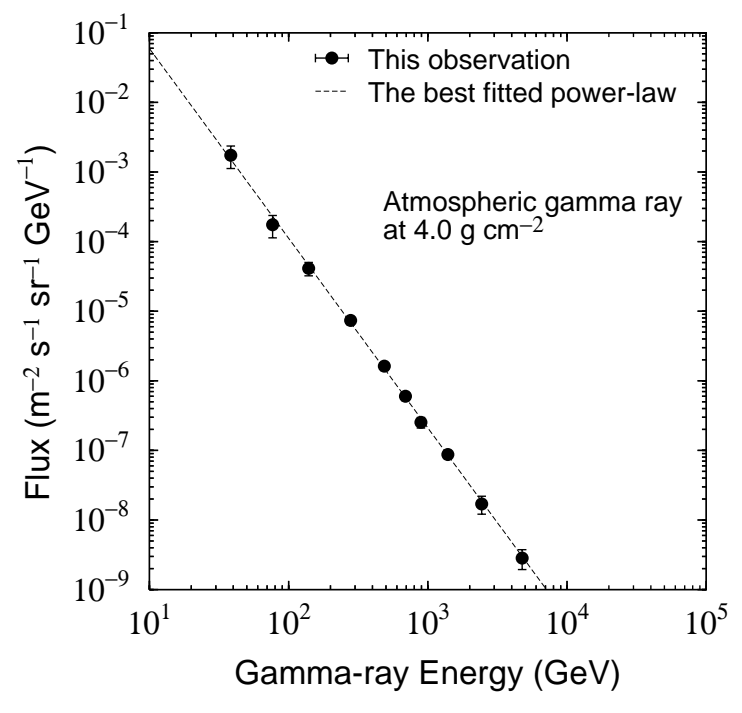

FIG. 6: The atmospheric gamma-ray spectrum observed at an altitude of $4.0 \mathrm{~g} \mathrm{~cm}^{-2}$. The dash line shows the best fit power-law function with an index of $-2.73 \pm 0.06$.

TABLE III: Atmospheric gamma-ray fluxes and raw number of gamma rays at $4.0 \mathrm{~g} \mathrm{~cm}^{-2}$

\begin{tabular}{cccc}
\hline \hline $\begin{array}{c}\text { Energy } \\
(\mathrm{GeV})\end{array}$ & $\begin{array}{c}\bar{E} \\
(\mathrm{GeV})\end{array}$ & $\begin{array}{c}\text { Raw } \\
\text { Number }\end{array}$ & $\begin{array}{c}\text { Flux } \\
\left(\mathrm{m}^{-2} \mathrm{~s}^{-1} \mathrm{sr}^{-1} \mathrm{GeV}^{-1}\right)\end{array}$ \\
\hline $30-50$ & $3.84 \times 10^{1}$ & 8 & $(1.74 \pm 0.62) \times 10^{-3}$ \\
$60-100$ & $7.68 \times 10^{1}$ & 8 & $(1.75 \pm 0.62) \times 10^{-4}$ \\
$100-200$ & $1.39 \times 10^{2}$ & 21 & $(4.12 \pm 0.90) \times 10^{-5}$ \\
$200-400$ & $2.79 \times 10^{2}$ & 59 & $(7.36 \pm 0.96) \times 10^{-6}$ \\
$400-600$ & $4.87 \times 10^{2}$ & 58 & $(1.62 \pm 0.21) \times 10^{-6}$ \\
$600-800$ & $6.91 \times 10^{2}$ & 59 & $(5.99 \pm 0.78) \times 10^{-7}$ \\
$800-1000$ & $8.93 \times 10^{2}$ & 34 & $(2.53 \pm 0.43) \times 10^{-7}$ \\
$1000-2000$ & $1.39 \times 10^{3}$ & 61 & $(8.70 \pm 1.11) \times 10^{-8}$ \\
$2000-3000$ & $2.44 \times 10^{3}$ & 12 & $(1.70 \pm 0.49) \times 10^{-8}$ \\
$3000-8000$ & $4.76 \times 10^{3}$ & 10 & $(2.84 \pm 0.90) \times 10^{-9}$ \\
\hline
\end{tabular}

\section{DECONVOLUTION OF PRIMARY PROTON SPECTRUM}

We assume that the primary protons at the top of atmosphere have a power-law spectrum of

$$
J_{p}(E) d E_{p}=N E_{p}^{-\gamma} d E_{p}
$$

As described below, the mean free path length of hadronic interactions and effective attenuation length of protons in the atmosphere are $\lambda_{p} \simeq 80 \mathrm{~g} \mathrm{~cm}^{-2}$ and $\Lambda_{p} \simeq$ $100 \mathrm{~g} \mathrm{~cm}^{-2}$ in the $\mathrm{TeV}$ region, respectively 35,36 . The flux of atmospheric gamma rays observed at $4.0 \mathrm{~g} \mathrm{~cm}^{-2}$ is represented by

$$
J\left(E_{\gamma}\right)=g\left(E_{\gamma}\right) \frac{1}{\lambda_{p}} \frac{\exp \left(-4.0 / \Lambda_{p}\right)-\exp \left(-4.0 \sigma_{0} / X_{0}\right)}{\sigma_{0} / X_{0}-1 / \Lambda_{p}},
$$

considering the absorption of gamma-rays in the atmosphere, where $g\left(E_{\gamma}\right)$ is a production spectrum of gammarays generated in collisions between protons and atmospheric nuclei.

In the energy range over $100 \mathrm{GeV}$, one can apply a scaling law in the first approximation for the production probability of $\pi^{0}$. The $\pi^{0}$ production rate in a single collision between protons and atmospheric nuclei is represented by

$$
f\left(E_{\pi^{0}} / E_{p}\right) d\left(E_{\pi^{0}} / E_{p}\right)
$$

assuming a scaling law, where $E_{\pi^{0}}$ is the energy of secondary $\pi^{0}$ and $E_{p}$ the energy of parent protons.

The production spectrum of $\pi^{0}$ generated in collisions between protons with a power-law spectrum and atmospheric nuclei is now given by

$$
\Pi\left(E_{\pi^{0}}\right) d E_{\pi^{0}}=\int_{E_{\pi^{0}}}^{\infty} d E_{p} E_{p}^{-\gamma} f\left(E_{\pi^{0}} / E_{p}\right) d\left(E_{\pi^{0}} / E_{p}\right)
$$

Putting

$$
Z_{p \pi^{0}}=\int_{0}^{1} x^{\gamma-1} f(x) d x
$$

where $x=E_{\pi^{0}} / E_{p}$, we have a simple formula of

$$
\Pi\left(E_{\pi^{0}}\right) d E_{\pi^{0}}=Z_{p \pi^{0}} E_{\pi^{0}}^{-\gamma} d E_{\pi^{0}}
$$

in which $Z_{p \pi^{0}}$ is the spectrum-weighted moment, called as $Z$ factor [37]. We derived a numerical value of $Z_{p \pi^{0}}$ for the case of power-law index $\gamma=2.75$, using the hadronic interaction models Dpmjet3 [38] and Fritiof V7.02 [39]. The results are shown in Fig. 7 Here we include the contribution of $\eta$ mesons to gamma rays. In implementing hadronic interaction models, we used a Monte Carlo simulator called COSMOS[30]. Although we adopted a scaling-law for the deconvolution of atmospheric gamma rays to primary protons, there is a weak energy dependence in the $Z$ factor of the formula (15). Therefore we need to correct this energy dependence. As for the hadronic interaction models used, there are several \% differences below $1 \mathrm{TeV}$ between Dpmjet3 and Fritiof V7.02, but above $1 \mathrm{TeV}$, the results from these two models agree well with each other.

The production spectrum of gamma rays from a single $\pi^{0}$ decay with energy $E_{\pi^{0}}$ is given by

$$
\frac{d n_{\gamma}}{d E_{\gamma}}=\frac{2}{E_{\pi^{0}}}
$$

Combining the formula (16) and (17), the energy spectrum of gamma rays from a single collision between protons and atmospheric nuclei is given by

$$
g(E)=\int_{E}^{\infty} \frac{d n_{\gamma}}{d E_{\gamma}} \Pi\left(E_{\pi^{0}}\right) d E_{\pi^{0}}=\frac{2 Z_{p \pi^{0}}}{\gamma} E^{-\gamma}
$$




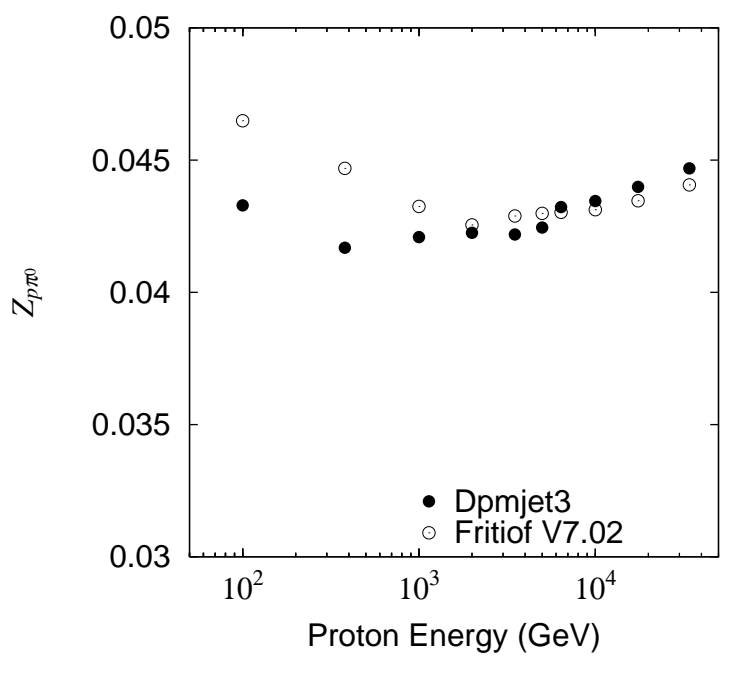

FIG. 7: Spectrum weighted moment $Z_{p \pi^{0}}$ of Dpmjet3 and Fritiof V7.02 for $p+$ Air $\rightarrow \pi^{0}+X$ with the parent proton energy, including the contribution of $\eta$ meson decay for gamma rays.

From equation (12) and (18), the energy spectrum of gamma rays from interactions of primary protons in the atmosphere is represented by

$$
J_{\gamma}(E)=\frac{2}{\gamma} Z_{p \pi^{0}} C_{\mathrm{atm}}^{p} N E^{-\gamma}
$$

where

$$
C_{\mathrm{atm}}^{p}=\frac{1}{\lambda_{p}} \frac{\exp \left(-x / \Lambda_{p}\right)-\exp \left(-\sigma_{0} x / X_{0}\right)}{\sigma_{0} / X_{0}-1 / \Lambda_{p}} .
$$

Using this formula, we can derive the primary proton spectrum from the observed atmospheric gamma rays. The interaction length of protons in the atmosphere is described by the expression $\lambda_{p}=A m_{p} / \sigma_{p}^{a i r}(A=14.5)$, where $A$ is the average mass number of atmospheric nuclei, $m_{p}$ is the mass of protons, and $\sigma_{p}^{a i r}$ is the cross section for proton-air interaction. We also derived the interaction length from the cross section given in references [35, 36]. We derived the attenuation length of protons in the atmosphere using the formula $\Lambda_{p}=$ $\lambda_{p} /\left(1-Z_{p p}-Z_{p n}\right)$. The spectrum-weighted moments of $Z_{p p}$ and $Z_{p n}$ are calculated using the hadronic interaction codes of Fritiof V7.02 and Dpmjet3. We present the interaction length $\lambda_{p}$ and attenuation length $\Lambda_{p}$ of protons for various energies in Fig. 8.

Here we need to include the contribution due to heavy primaries. We corrected for the effects of heavier nuclei such as $\mathrm{He}, \mathrm{C}, \mathrm{N}$, and $\mathrm{O}$. The flux of heavier nuclei is $\sim 6.4 \%$ of the flux of protons at the same energy per nucleon for $\mathrm{He}$, and $\sim 0.57 \%$ for $\mathrm{C}, \mathrm{N}, \mathrm{O}$, based on the JACEE and RUNJOB observations [12, 13, 14]. Since interaction lengths for $\mathrm{He}$ and $\mathrm{C}, \mathrm{N}, \mathrm{O}$ components are $\lambda_{\mathrm{He}} / \lambda_{p}=0.56$ for $\mathrm{He}, \lambda_{N} / \lambda_{p}=0.29$ for $\mathrm{C}, \mathrm{N}, \mathrm{O}$, us-

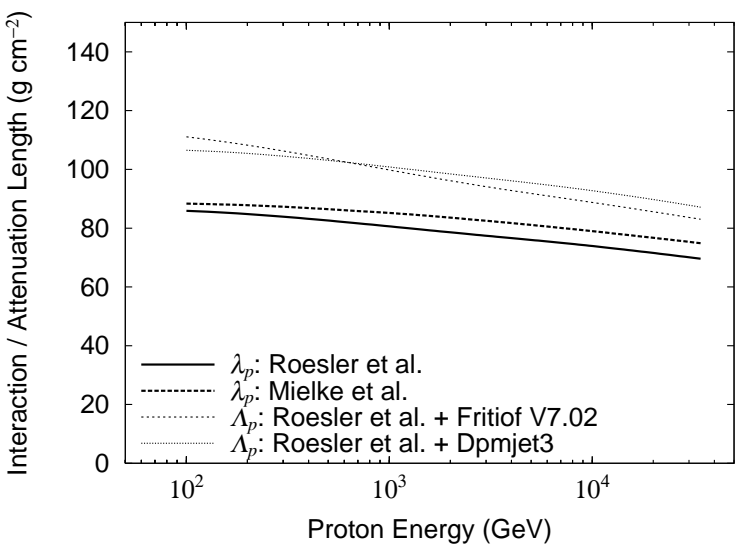

FIG. 8: Energy dependence of mean free path lengths of hadronic interactions $\lambda_{p}$ and attenuation lengths $\Lambda_{p}$ of protons in the atmosphere [35, 36].

ing the Hagen-Watts formula [4], atmospheric interaction contributions are $C_{\mathrm{atm}}^{\mathrm{He}} / C_{\mathrm{atm}}^{p}=1.73$ for $\mathrm{He}$, and $C_{\mathrm{atm}}^{N} / C_{\mathrm{atm}}^{p}=3.35$ for $\mathrm{C}, \mathrm{N}, \mathrm{O}$ from the formula (20). According to Dpmjet3, the spectrum-weighted moments are given by $Z_{H e \pi^{0}} / Z_{p \pi^{0}}=2.06$ for He and $Z_{N \pi^{0}} / Z_{p \pi^{0}}=$ 4.97 for C,N,O. Therefore, using formula (19), the flux of heavier nuclei can be converted to an equivalent proton flux as follows:

$$
\begin{aligned}
\frac{J_{H e}+J_{C N O}}{J_{p}} & =0.064 \frac{C_{\mathrm{atm}}^{H e}}{C_{\mathrm{atm}}^{p}} \frac{Z_{H e \pi^{0}}}{Z_{p \pi^{0}}}+0.0057 \frac{C_{\mathrm{atm}}^{N}}{C_{\mathrm{atm}}^{p}} \frac{Z_{N \pi^{0}}}{Z_{p \pi^{0}}} \\
& =0.32 .
\end{aligned}
$$

Therefore, the proton flux from the gamma rays is multiplied by $1 / 1.32=0.76$. This correction factor is almost same as the results of an independent-nucleon model for heavier nuclei as follows: $4 \times 0.064+14 \times 0.0057=0.34$, that is $1 / 1.34=0.75$. The uncertainty of the correction factor is mainly from the uncertainties of the currently observed flux of heavy primary [12, 13, 14], and is estimated to be no more than $\sim 5 \%$.

We also corrected for the minor contributions of gamma rays from $\eta$ and $K$ mesons, using Dpmjet3 results. The contribution from $\eta$ mesons is $\sim 0.16$ as large as from $\pi^{0}$ mesons. The correction for $\eta$ mesons is included in the calculation of $Z_{p \pi^{0}}$. The contribution of gamma rays from $K^{0}$ mesons is $\sim 0.03$ as large as those from $\pi^{0}$ mesons. To correct this contribution, we multiplied the proton flux by the correction factor of $1 / 1.03=0.97$.

As shown from equation (18), one proton produces gamma-rays of $(\gamma / 2)\left(1 / Z_{p \pi^{0}}\right)$. Hence, the energy of the corresponding parent protons is $C_{\mathrm{E}}=\left(2 Z_{p \pi^{0}} / \gamma\right)^{-1 /(\gamma-1)}$ times of that of the gamma rays. Thus we can presume that atmospheric gamma rays are on average produced from primary protons that have $C_{\mathrm{E}}$ times higher energy than the daughter gamma rays. $C_{\mathrm{E}}$ is $\sim 7.2$ for Dpmjet3 with $\gamma=2.75$. 
In this way, we derived the deconvolved primary proton spectrum as

$$
\begin{aligned}
J_{p}\left(E_{p}\right)=\frac{\gamma}{2} \frac{1}{Z_{p \pi^{0}}} \lambda_{p} & \frac{\sigma_{0} / X_{0}-1 / \Lambda_{p}}{\exp \left(-x / \Lambda_{p}\right)-\exp \left(-\sigma_{0} x / X_{0}\right)} \\
& \times 0.76 \times 0.97 \times C_{\mathrm{E}}^{-\gamma} J_{\gamma}\left(E_{\gamma}\right),
\end{aligned}
$$

where $E_{p}=C_{\mathrm{E}} E_{\gamma}$. Using this formula, we obtained the primary cosmic-ray proton spectrum, using the Dpmjet3 hadronic interaction model, from the atmospheric gamma-ray spectrum at $4.0 \mathrm{~g} \mathrm{~cm}^{-2}$ as presented in Fig. 9] Figure 9 shows our deconvolved proton spectrum with the directly observed proton spectra at high altitude obtained by other groups [6, 7, 9, 11, 12, 13, 15, 41]. Our proton flux with Dpmjet3 (open circles) is $~ 20 \%$ larger than the extrapolation of the BESS, AMS-01, and BESS$\mathrm{TeV}$ data.

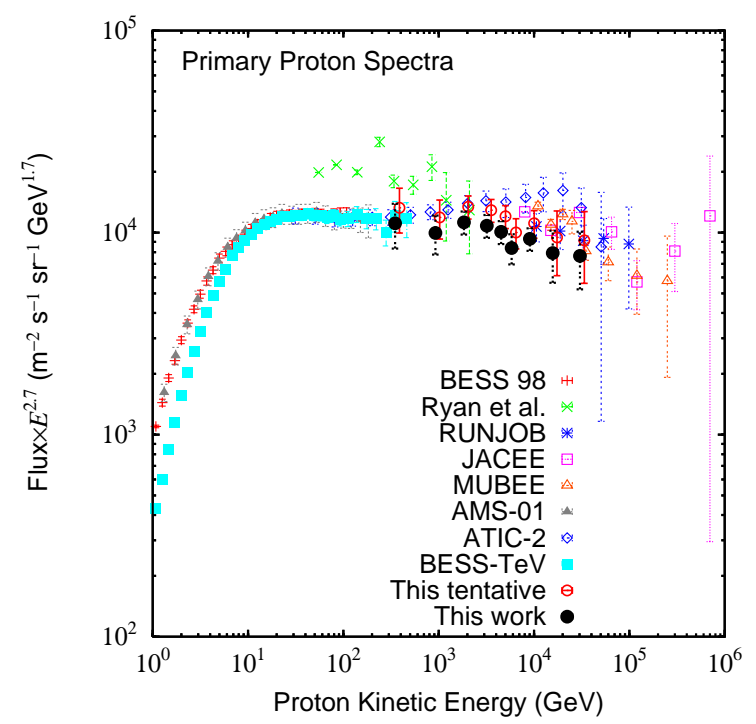

FIG. 9: The proton spectra deconvolved from the atmospheric gamma-ray spectrum, compared with the spectra observed by other groups $[6,17,9,11,12,13,15,41]$. Open circles show the proton spectrum derived from the gamma-ray spectrum with Dpmjet3. Solid circles show our final proton spectrum derived with $20 \%$ larger $Z_{\pi^{0}}$ than that of Dpmjet3.

The uncertainties in deriving the flux of primary protons from atmospheric gamma-ray flux come mainly from the hadronic interaction models, while the muon flux estimated from the gamma-ray flux is less sensitive to the treatment of hadronic interactions, as shown in section IV] and appendix B] For hadronic interactions, there are uncertainties in factors such as the interaction length of protons in the atmosphere and the production rate of pions. The former has an uncertainty of several $\%$ as shown in Fig. 8 and the uncertainty of the latter is estimated to be much larger, $\sim 20-25 \%[37$. Therefore, the main systematic error contribution in the estimation of the proton flux from the gamma-ray flux comes from the uncertainty of the $Z$ factor for the production rate of pions. Sanuki et al. (2006) pointed out that the muon flux measured with the BESS-TeV and $\mathrm{L} 3+\mathrm{C}$ above $100 \mathrm{GeV}$ is $\sim 20 \%$ larger than the calculated muon flux with Dpmjet3, and that the muon charge ratio $\left(\mu^{+} / \mu^{-}\right)$is inconsistent with that predicted by Dpmjet3. They suggested that in the energy range of $100 \mathrm{GeV}$ to $10 \mathrm{TeV}$ the production rates of charged pions and kaons are $\sim 20 \%$ larger than that by Dpmjet3 21. They also proposed that Dpmjet3 can be modified in a phenomenological way, with an assumption based on the quark model, to increase by $\sim 20 \%$ above $\sim 100 \mathrm{GeV}$ the $\mathrm{Z}$ factors of secondary particles, accommodating the calculated muon charge ratio to the observed one.

Referring to their modified model, we adopted $20 \%$ larger $Z_{\pi^{0}}$ than that of Dpmjet3 and derived the proton flux from the gamma-ray flux using formula (22). The energy of the corresponding parent protons is $C_{\mathrm{E}} \simeq 6.5$ times of that of the gamma rays. The derived proton spectrum (solid circles) is presented in Fig. 9] and is well represented by

$$
\begin{array}{r}
J_{p}(E)=(5.4 \pm 1.2) \times 10^{-2}(E / 100 \mathrm{GeV})^{-2.79 \pm 0.06} \\
\left(\mathrm{~m}^{-2} \mathrm{~s}^{-1} \mathrm{sr}^{-1} \mathrm{GeV}^{-1}\right)
\end{array}
$$

in the energy range from $200 \mathrm{GeV}$ to $50 \mathrm{TeV}$. The flux values are also summarized in Table IV To derive the proton spectrum, we assumed a power-law index of -2.75 . The fitted indexes are -2.73 for the gamma rays and -2.79 for the protons, slightly steeper than the gamma rays, because of the weak energy dependences of $\mathrm{Z}$ factors and interaction lengths, as shown in Fig. 7 and Fig. 8 The differences of power-law indexes from -2.75 cause $\sim 5 \%$ differences for the proton flux, which are much smaller than the main uncertainty of hadronic interaction models of $\sim 20 \%$.

Our deconvolved proton spectrum above $10 \mathrm{TeV}$ is somewhat smaller than the JACEE and MUBEE data, and agrees well with the RUNJOB data. The ATIC-2 data show much higher flux than our proton flux. Below $10 \mathrm{TeV}$, our proton spectrum is consistent with the extrapolated fluxes with the BESS, AMS-01, and BESSTeV data.

\section{ESTIMATION OF HIGH-ENERGY MUON SPECTRUM AT HIGH ALTITUDE}

Atmospheric muons mainly come from the decay of charged pions and kaons. We discuss the contributions of pions and kaons for muon production.

In the first approximation, the same number of $\pi^{-}$and $\pi^{+}$mesons are produced as $\pi^{0}$. Therefore, the production spectrum of charged pions can be estimated from that of atmospheric gamma rays through $\pi^{0}$, independent of primary cosmic rays, such as $F_{\pi^{ \pm}} \simeq 2 F_{\pi^{0}} \simeq \gamma F_{\gamma}$, where $F_{\pi^{ \pm}}, F_{\pi^{0}}$ and $F_{\gamma}$ are the production spectrum of $\pi^{ \pm}$, $\pi^{0}$ and atmospheric gamma rays per unit depth, respectively. In more detail, the production ratio of charged 
TABLE IV: Deconvolved primary proton fluxes

\begin{tabular}{cc}
\hline \hline $\begin{array}{c}\bar{E} \\
(\mathrm{GeV})\end{array}$ & $\begin{array}{c}\text { Flux } \\
\left(\mathrm{m}^{-2} \mathrm{~s}^{-1} \mathrm{sr}^{-1} \mathrm{GeV}^{-1}\right)\end{array}$ \\
\hline $3.49 \times 10^{2}$ & $(1.52 \pm 0.38) \times 10^{-3}$ \\
$9.23 \times 10^{2}$ & $(9.83 \pm 2.15) \times 10^{-5}$ \\
$1.84 \times 10^{3}$ & $(1.72 \pm 0.22) \times 10^{-5}$ \\
$3.20 \times 10^{3}$ & $(3.73 \pm 0.48) \times 10^{-6}$ \\
$4.52 \times 10^{3}$ & $(1.36 \pm 0.18) \times 10^{-6}$ \\
$5.83 \times 10^{3}$ & $(5.72 \pm 0.97) \times 10^{-7}$ \\
$9.04 \times 10^{3}$ & $(1.94 \pm 0.25) \times 10^{-7}$ \\
$1.57 \times 10^{4}$ & $(3.72 \pm 1.07) \times 10^{-8}$ \\
$3.03 \times 10^{4}$ & $(6.07 \pm 1.92) \times 10^{-9}$ \\
\hline
\end{tabular}

pions to neutral pions is calculated to be 1.7 using a hadronic interaction model of Dpmjet3.

Since the $\pi^{ \pm}$lifetime for decay to muons is $2.6 \times$ $10^{-8} \mathrm{sec}, \pi^{ \pm}$mesons above $\sim 100 \mathrm{GeV}$ travel more than $\sim 5 \mathrm{~km}$ on the average and may interact with atmospheric nuclei before decay. On the other hand, charged kaons have larger mass, $493.6 \mathrm{MeV}$, and shorter life time, $1.2 \times 10^{-8}$ sec. Therefore, although atmospheric muons are mainly produced by the decay of $\pi^{ \pm}$, the relative contribution of kaons to the muon flux increases with increasing energy above $\sim 100 \mathrm{GeV}$ 37].

Hence, on the basis of the gamma-ray flux from $\pi^{0}$, we can derive the flux of muons from $\pi^{ \pm}$and $K$, correcting for muon decay and the flux of gamma rays from $\eta$ and $K^{0}$ mesons. The muon flux is represented as the following formula of

$$
J_{\mu}=S_{\mu}\left(f_{\pi^{ \pm}}+f_{K}\right) \frac{J_{\gamma}}{1+f_{\eta}},
$$

where $S_{\mu}$ is the survival probability of muons, $f_{\pi^{ \pm}}$is the ratio of the muon flux from $\pi^{ \pm}$to the gamma-ray flux from $\pi^{0}, f_{K}$ is a ratio of the muon flux from kaons to the gamma-ray flux from $\pi^{0}$, and $f_{\eta}$ is the ratio of the gamma-ray flux from $\eta+K$ to the gamma-ray flux from $\pi^{0}$. We derived the production ratio of $K^{ \pm} / \pi^{ \pm}$using the hadronic interaction model of Dpmjet3. Figure 10 shows $S_{\mu}, f_{\pi}, f_{K}$, and $f_{\eta}$ as a function of muon energy. The detailed derivation for these parameters is given in appendix B

We transformed the observed atmospheric gamma-ray spectrum to the muon spectrum using the formula (24), and results are presented in Fig. [1] and Table $\nabla$ In Fig. [11] we compare our estimated $\mu^{+}+\mu^{-}$spectrum at $4.0 \mathrm{~g} \mathrm{~cm}^{-2}$ with that of $\mu^{+}$and $\mu^{-}$observed by BESS and HEAT group 18, 19 as representative values, because the results by the BESS, HEAT, MASS and CAPRICE are consistent with each other within statistical errors. Since the BESS and HEAT observations are performed at altitudes of $4.5 \mathrm{~g} \mathrm{~cm}^{-2}$ and $5.6 \mathrm{~g} \mathrm{~cm}^{-2}$ respectively, the coefficients of $4.0 / 4.5$ and $4.0 / 5.6$ are multiplied to obtain the muon flux at $4.0 \mathrm{~g} \mathrm{~cm}^{-2}$ for the comparison. As shown in Fig. [1] our flux is just on the line

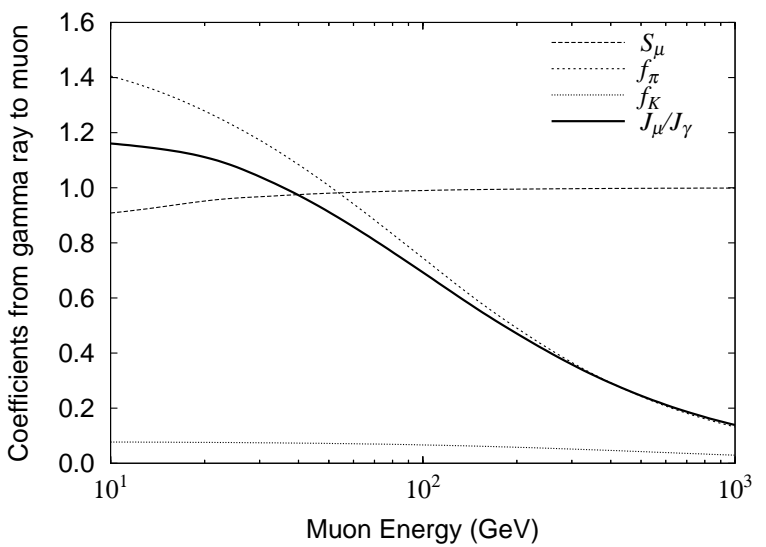

FIG. 10: Coefficients describing the relation between the muon flux $J_{\mu}$ and the gamma-ray flux $J_{\gamma} . J_{\mu}=S_{\mu}\left(f_{\pi^{ \pm}}+\right.$ $\left.f_{K}\right) J_{\gamma} /\left(1+f_{\eta}\right)$, where $f_{\eta}=0.16$. $S_{\mu}$ is a survival probability of $\mu^{ \pm}$. $f_{\pi^{ \pm}}, f_{K}$, and $f_{\eta}$ are flux ratios of $\pi^{ \pm} \rightarrow \mu^{ \pm}$to $\pi^{0} \rightarrow \gamma$, $K^{ \pm} \rightarrow \mu^{ \pm}$to $\pi^{0} \rightarrow \gamma$, and $\eta, K^{0} \rightarrow \gamma$ to $\pi^{0} \rightarrow \gamma$, respectively. See text in detail.

of extrapolation of each datum of the BESS and HEAT experiments and gives consistent values within statistical errors, although the energy region of our gamma rays is almost one order of magnitude higher than that of the directly observed muons.

To calculate the atmospheric neutrino flux at energies above $\sim 100 \mathrm{GeV}$ with better accuracy, muon flux data above $\sim 100 \mathrm{GeV}$ measured at high altitude would be very useful for the calibration of the hadronic interaction model. However, we have no direct observations of muons above $\sim 100 \mathrm{GeV}$ at high altitude. Our estimated muon flux gives useful information for this purpose.

TABLE V: Estimated atmospheric $\mu^{+}+\mu^{-}$fluxes at $4.0 \mathrm{~g} \mathrm{~cm}^{-2}$

\begin{tabular}{cc}
\hline \hline $\begin{array}{c}\bar{P} \\
(\mathrm{GeV} / \mathrm{c})\end{array}$ & $\begin{array}{c}\text { Flux } \\
\left(\mathrm{m}^{-2} \mathrm{~s}^{-1} \mathrm{sr}^{-1}(\mathrm{GeV} / \mathrm{c})^{-1}\right)\end{array}$ \\
\hline $3.85 \times 10^{1}$ & $(1.70 \pm 0.61) \times 10^{-3}$ \\
$7.69 \times 10^{1}$ & $(1.36 \pm 0.48) \times 10^{-4}$ \\
$1.39 \times 10^{2}$ & $(2.43 \pm 0.53) \times 10^{-5}$ \\
$2.79 \times 10^{2}$ & $(2.80 \pm 0.37) \times 10^{-6}$ \\
$4.87 \times 10^{2}$ & $(3.97 \pm 0.52) \times 10^{-7}$ \\
$6.91 \times 10^{2}$ & $(1.10 \pm 0.14) \times 10^{-7}$ \\
\hline
\end{tabular}

\section{SUMMARY}

We have observed atmospheric gamma rays at several $\mathrm{g} \mathrm{cm}^{-2}$ altitude with emulsion chambers. Precise measurements of the atmospheric gamma-ray spectrum from hadronic interactions have been performed with the 


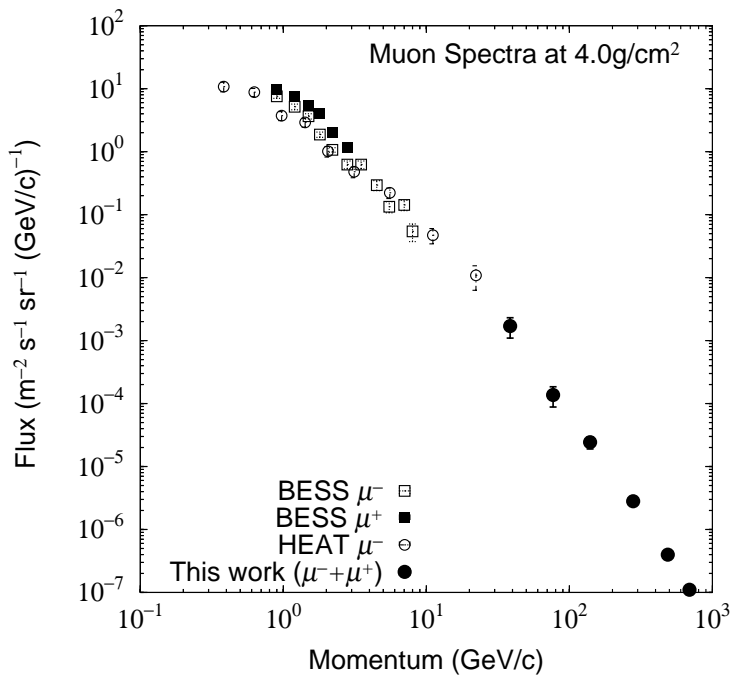

FIG. 11: Comparison of muon spectra estimated by our gamma-ray observation with those observed by BESS 19 and HEAT 18]. The altitudes are normalized at $4.0 \mathrm{~g} \mathrm{~cm}^{-2}$. In the direct observations charged muons are measured individually, and in our estimation all charged muons are included together.

largest $S \Omega T$ among all existing measurements in the energy range $30 \mathrm{GeV}$ to $8 \mathrm{TeV}$. To obtain the atmospheric gamma-ray flux at $4.0 \mathrm{~g} \mathrm{~cm}^{-2}$ from the observed data at each altitude, we took into account several correction factors such as gamma-ray detection efficiency, enhancement due to energy resolution, bremsstrahlung gammaray flux from primary electrons, and altitude conversion to $4.0 \mathrm{~g} \mathrm{~cm}^{-2}$. Although some electronic detectors have uncertainty in the determination of their geometrical factor $S \Omega_{\gamma}$, that of the emulsion chambers can be estimated very accurately because of the precise determination of the track location and the simple configuration of the detector. Thus, the uncertainties of these correction factors and $S \Omega_{\gamma}$ are relatively smaller than the statistical errors. We deconvolved primary proton spectrum in the $200 \mathrm{GeV}$ - $50 \mathrm{TeV}$ from our gamma-ray spectrum in a reliable way, assuming a single interaction of each proton with an atmospheric nucleus. The main uncertainty in the deconvolution comes from hadronic interaction models, and we referred to a phenomenologically modified model in Dpmjet3 21]. While in the energy range from $100 \mathrm{GeV}$ to $10 \mathrm{TeV}$ accurate data are missing in the currently observed proton spectra, our estimated proton spectrum fills this gap. Our derived proton flux is consistent with the other observed data in the overlapping region. This may also indicate the validity of the hadronic interaction model in the TeV region proposed by Sanuki et al. 21]. From the gamma-ray spectrum, we also deconvolved the atmospheric muon spectrum, which is consistent with direct muon observations below $10 \mathrm{GeV}$.

\section{Acknowledgments}

We sincerely thank the crews of the SBC of JAXA/ISAS and the NSBF for the successful balloon flights. We thank K.Kasahara and M.Honda for their helpful discussions and help in implementing hadronic interaction codes. We are also grateful to R.J.Wilkes for his careful reading of the manuscript.

\section{APPENDIX A: ENHANCEMENT FACTOR OF FLUX BY ENERGY RESOLUTION}

Defining $E_{0}$ as the incident gamma-ray energy and $\sigma$ as the standard deviation of energy determination, the observed energy $E$ has an uncertainty given by the Gaussian probability function

$$
P\left(E-E_{0}\right)=\frac{1}{\sqrt{2 \pi} \sigma} \exp \left(-\left(E-E_{0}\right)^{2} /\left(2 \sigma^{2}\right)\right)
$$

As the gamma-ray spectrum is a power-law function of $E_{0}^{-\gamma} d E_{0}$, the enhancement factor is given by

$$
C_{\mathrm{enh}}=\frac{1}{E^{-\gamma}} \int_{0}^{\infty} E_{0}^{-\gamma} P\left(E-E_{0}\right) d E_{0}
$$

In the case of constant energy resolution $\sigma / E_{0}=$ const., it is well represented by the series form of

$$
C_{\mathrm{enh}}=1+\frac{(\gamma-1)(\gamma-2)}{2}\left(\frac{\sigma}{E_{0}}\right)^{2}+\cdots,
$$

independent of the gamma-ray energy. The exact solution can be presented by a hypergeometric function. In the case of energy resolution $\sigma / E_{0}=15 \%$, with $\gamma=2.75$, this enhancement factor is 1.01. In emulsion chambers, the energy resolution is well represented by the form of (41). Using numerical integration with the formula (A1), (A2), and (4), we derived the enhancement factor $C_{\text {enh }}$ for each gamma-ray energy bin and for each emulsion chamber. $C_{\text {enh }}$ has values from 1.00 to 1.06 .

\section{APPENDIX B: CONTRIBUTION OF PIONS AND KAONS FOR MUON PRODUCTION}

\section{Decay factor $B$ for each particle}

In the case of an isothermal atmosphere, secondary particles produced at a depth of $x \mathrm{~g} \mathrm{~cm}^{-2}$ arrive at a depth of $x_{0} \mathrm{~g} \mathrm{~cm}^{-2}$ without decay with a survival probability of $\left(x / x_{0}\right)^{(B / E)}$ per unit depth, neglecting the ionization loss of the particles, where $B$ is the decay factor and $E$ is the energy for each particle [42]. We define the decay factor $B$ as $B=H m /(\tau c)$, where $H$ is the scale height of the atmosphere, $m$ is particle mass, $\tau$ is particle decay lifetime, and $c$ is light speed. Setting scale height $H$ to be $6.3 \mathrm{~km}$ at balloon altitude, the decay factors are $B_{\mu}=1.0 \mathrm{GeV}$ for muons, $B_{\pi}=112.7 \mathrm{GeV}$ for pions, and $B_{K}=838.5 \mathrm{GeV}$ for kaons. 


\section{Decay of muons}

At balloon altitudes of several $\mathrm{g} \mathrm{cm}^{-2}$ residual overburden, atmospheric muons are produced at a rate proportional to the transverse depth $x$, because the interaction length for cosmic rays on atmospheric nuclei is much larger than the atmospheric depth of several $\mathrm{g} \mathrm{cm}^{-2}$. Therefore, the survival probability $S_{\mu}$ of muons without decay is given by

$$
S_{\mu}=\frac{1}{x_{0}} \int_{0}^{x_{0}}\left(\frac{x}{x_{0}}\right)^{B_{\mu} / E} d x=\frac{E}{B_{\mu}+E} .
$$

\section{Decay of pions}

The flux of $\pi^{ \pm}$decaying per unit depth is obtained by multiplying $B_{\pi} / E$ into the formula (B1) and normalizing by the production rate as

$$
\left(\frac{B_{\pi}}{B_{\pi}+E}\right) F_{\pi}
$$

Hence the flux of $\pi^{ \pm}$decaying until a depth of $x_{0} \mathrm{~g} \mathrm{~cm}^{-2}$ is given by

$$
x_{0}\left(\frac{B_{\pi}}{B_{\pi}+E}\right) F_{\pi}=0.85 \gamma\left(\frac{B_{\pi}}{B_{\pi}+E}\right) J_{\gamma},
$$

where $J_{\gamma}$ is the atmospheric gamma-ray flux at $x_{0} \mathrm{~g} \mathrm{~cm}^{-2}$ and given by $J_{\gamma}=x_{0} F_{\gamma}$ from the relation of $F_{\pi}=$ $1.7 F_{\pi^{0}}=0.85 \gamma F_{\gamma}$. Here, the production ratio of $\pi^{ \pm}$to $\pi^{0}$ is calculated to be 1.7 using the hadronic interaction code Dpmjet3.

As described in reference [42], the flux of muons produced from the decay of pions at a depth of $x \mathrm{~g} \mathrm{~cm}^{-2}$, per unit depth, is given by

$$
\frac{m_{\pi}^{2}}{m_{\pi}^{2}-m_{\mu}^{2}} \int_{E_{-}}^{E_{+}}\left(\frac{B_{\pi}}{B_{\pi}+E_{\pi}}\right) F_{\pi}\left(E_{\pi}\right) \frac{d E_{\pi}}{E_{\pi}}
$$

where $E_{+}$and $E_{-}$show the upper and lower limits of the energy of pions with rest mass $m_{\pi}$ that produce muons with rest mass $m_{\mu}$ energy $E_{\mu} . E_{+}$and $E_{-}$are given by

$$
E_{+}=\left(\frac{m_{\pi}}{m_{\mu}}\right)\left(\frac{E_{\mu} E_{\mu}^{*}+p_{\mu} p_{\mu}^{*} c^{2}}{m_{\mu} c^{2}}\right) \simeq\left(\frac{m_{\pi}}{m_{\mu}}\right)^{2} E_{\mu}=\frac{E_{\mu}}{r_{\pi}}
$$

and

$$
E_{-}=\left(\frac{m_{\pi}}{m_{\mu}}\right)\left(\frac{E_{\mu} E_{\mu}^{*}-p_{\mu} p_{\mu}^{*} c^{2}}{m_{\mu} c^{2}}\right) \simeq E_{\mu}
$$

where $r_{\pi}$ is $\left(m_{\mu} / m_{\pi}\right)^{2}=0.5733, p_{\mu}$ is momentum of pion, and the notation ' $*$ ' means a static system.
In the case of the complete decay of pions, the flux of muons per unit depth at a depth of $x_{0} \mathrm{~g} \mathrm{~cm}^{-2}$ is given by

$$
\begin{aligned}
& x_{0} \frac{m_{\pi}^{2}}{m_{\pi}^{2}-m_{\mu}^{2}} \int_{E_{-}}^{E_{+}} F_{\pi}\left(E_{\pi}\right) \frac{d E_{\pi}}{E_{\pi}} \\
& \quad=\frac{x_{0}}{1-r_{\pi}} \int_{E_{\mu}}^{E_{\mu} / r_{\pi}} E_{\pi}^{-\gamma} \frac{d E_{\pi}}{E_{\pi}}=x_{0} \frac{1-r_{\pi}^{\gamma}}{1-r_{\pi}} \frac{E_{\mu}^{-\gamma}}{0.85 \gamma} .
\end{aligned}
$$

As a result, the ratio of the flux of muons from the decay of charged pions to the flux of gamma rays from the decay of neutral pions is given by

$$
f_{\pi}\left(E_{\mu}\right)=\frac{0.85 \gamma E_{\mu}^{\gamma}}{1-r_{\pi}^{\gamma}} \int_{E_{\mu}}^{E_{\mu} / r_{\pi}}\left(\frac{B_{\pi}}{B_{\pi}+E_{\pi}}\right) E_{\pi}^{-\gamma} \frac{d E_{\pi}}{E_{\pi}}
$$

\section{Decay of kaons}

Although muons are mainly produced from the decay of charged pions, there is a minor contribution from kaons [37]. While there are $K^{ \pm}, K_{S}^{0}$ and $K_{L}^{0}$, only the charged kaons decay directly to muons via $K^{ \pm} \rightarrow \mu^{ \pm}+\nu$. For the production of muons the contribution of kaons can be treated in the same way as pions. We derive the relation between kaons and gamma rays through the production ratio of $K^{ \pm} / \pi^{ \pm}$. The production ratio of $K^{ \pm} / \pi^{ \pm}$is 0.14 in the parent proton energy of $200 \mathrm{GeV}-40 \mathrm{TeV}$ according to the hadronic interaction code in Dpmjet3. The decay mode $K^{ \pm} \rightarrow \mu^{ \pm}$dominates (63\% of decays) among the charged kaon decay mode [43]. As a result, we can obtain the ratio of the flux of muons from kaons to the flux of gamma rays from neutral pions as

$$
\begin{aligned}
f_{K}\left(E_{\mu}\right)=0.14 \times 0.63 \times \frac{0.85 \gamma E_{\mu}^{\gamma}}{1-r_{K}^{\gamma}} \\
\int_{E_{\mu}}^{E_{\mu} / r_{K}}\left(\frac{B_{K}}{B_{K}+E_{K}}\right) E_{K}^{-\gamma} \frac{d E_{K}}{E_{K}},
\end{aligned}
$$

where $r_{K}$ is $\left(m_{\mu} / m_{K}\right)^{2}=0.0459$. Although there is a $K^{ \pm} \rightarrow \pi^{ \pm}$decay mode (21\% of decays), the contribution of $K^{ \pm} \rightarrow \pi^{ \pm} \rightarrow \mu^{ \pm}$is less than $\sim 1 \%$ for the total muon flux.

Since kaons have larger rest mass and shorter life time, the decay factor of kaons, $B_{K}=838.5 \mathrm{GeV}$, becomes larger than that of pions, $B_{\pi}=112.7 \mathrm{GeV}$. Therefore, the contribution of kaons continues into a higher energy region than pions. 
[3] T. Kobayashi et al., Proc. of 26th ICRC (Salt Lake) 2, 61 (1999).

[4] K. Kasahara et al., Phys. Rev. D 66, 052004 (2002).

[5] Y. Takahashi et al., Proc. of 24th ICRC (Roma) 2, 451 (1995).

[6] T. Sanuki et al., Astrophys. J. 545, 1135 (2000).

[7] M. Aguilar et al., Phys. Rep. 366/6, 331 (2002).

[8] M. Boezio et al., Astropart. Phys. 19, 583 (2003).

[9] S. Haino et al., Phys. Lett. B 594, 35 (2004).

[10] N. Grigorov et al., Can. J. Phys. 46, 687 (1968).

[11] M. Ryan et al., Phys. Rev. Lett. 28, 985 (1972).

[12] K.Asakimori et al., Astrohpys. J. 502, 278 (1998).

[13] A.V.Apanasenko et al., Astrop. Phys. 16, 13 (2001).

[14] V.A.Derbina et al., Astrophys. J. 628, L41 (2005).

[15] J. Wefel et al., Proc. of 29th ICRC (Pune) in press, 2005 (2005).

[16] A. Codino et al., J. Phys. G. 23, 1751 (1997).

[17] T. Francke et al., Proc. of 26th ICRC (Salt Lake) 2, 80 (1999).

[18] J. Beatty et al., Phys. Rev. D 70, 092005 (2004).

[19] T. Sanuki et al., Proc. of 27th ICRC (Hamburg) p. 950 (2001).

[20] K. Abe et al., Phys. Lett. B 564, 8 (2003).

[21] T. Sanuki et al. (2006), in preparation.

[22] Y. Sato et al., Proc. of 28th ICRC (Tsukuba) 4, 1821 (2003).

[23] K. Tang, Astrophys. J. 278, 881 (1984).

[24] C. Grimani et al., Astron. Astrophys. 392, 287 (2002).
[25] M. Boezio et al., Astrophys. J. 532, 653 (2000).

[26] M. Duvernois et al., Astrohpys. J. 559, 296 (2001).

[27] S. Torii et al., Astrohpys. J. 559, 973 (2001).

[28] T. Kobayashi et al., Nuovo Ciment 104A, 1753 (1991).

[29] E. Eskut et al., Nucl. Instr. Meth. A 401, 7 (1997).

[30] K. Kasahara, http://eweb.b6.kanagawau.ac.jp/ kasahara/.

[31] J. Nishimura, Handbuch der Physik XLVI/2, 1 (1967).

[32] S. Hunber et al., Astrophys. J. 481, 205 (1997).

[33] P. Sreekumar et al., Astrophys. J. 494, 523 (1998).

[34] I. A.Strong and O.Reimer, Astrophys. J. 613, 956 (2004).

[35] H. Mielke et al., J. Phys. G 20, 637 (1994).

[36] R. E. S. Roesler and J. Ranft, Proc. of 27th ICRC (Hamburg) p. 439 (2001).

[37] T. Gaisser and M. Honda, Ann. Rev. Nucl. Part. Sci. 52, $153(2002)$.

[38] R. E. S. Roesler and J. Ranft, Report No. SLCA-PUB8740, hep-ph/0012252, Proceeding of Monte Carlo 2000 72, 23 (2000).

[39] H. Pi, Comput. Phys. Commun. 71, 173 (1992).

[40] Y. Kawamura et al., Phys. Rev. D 40, 729 (1989).

[41] V. Zatsepin et al., Proc. of 23rd ICRC (Calgary) 2, 13 (1993).

[42] S. Hayakawa, Cosmic Ray Physics (Wiley-Interscience, 1969).

[43] S. Eidelman et al., Phys. Lett. B 592, 1 (2004). 